\title{
AMPLITUDE EQUATIONS FOR SPDES: APPROXIMATE CENTRE MANIFOLDS AND INVARIANT MEASURES
}

\author{
DIRK BLÖMKER * AND MARTIN HAIRER †
}

\begin{abstract}
We review recent results on the approximation of transient dynamics of SPDEs by amplitude equations. As an application we derive the flow along an approximate centre manifold, and we study the dynamics of random fixed points. To discuss the long-time behaviour we give an approximation result for invariant measures.
\end{abstract}

Key words. Amplitude equation, SPDE, separation of time scales, approximate centre manifold, bifurcation, multiple scale analysis, invariant measures

AMS(MOS) subject classifications. 60H10, 60H15.

1. Introduction. In systems near a change of stability, the essential dynamics is given by the evolution of the dominant eigenfunctions of the linearisation, and the effect of small noise is visible if the strength of the noise is comparable to the size of the linear instability. It is of course well-known how to linearise nonlinear equations to get a linear equation describing the essential stochastic dynamics close to an equilibrium. In this article we will also take into account the effect of nonlinearities on weakly unstable systems, allowing to describe the essential dynamics for much larger radii than the linearisation. Actually, we will see that under weak non-degeneracy conditions, this even allows to describe the long-time behaviour of the system under consideration, i.e. the invariant measures of the original system and the approximating system are close.

We also describe several byproducts of this method. One result is the existence of an approximate centre manifold around a weakly (un)stable equilibrium (cf. Theorem 4.1). This is a deterministic vector space on which the stochastic flow can be described by a suitable approximation. We also give results for dynamics of random attractors.

Our main tool is the approximation by amplitude equations. This theory is mathematically very well understood in the deterministic case, even for extended systems [12, 13, 34, 39]. It was first rigorously used for stochastic systems in [5] and later extended in $[4,7,8]$. We give a survey of this method, together with new results on the dynamics of random attractors and approximate centre manifolds.

The general methodology follows two main steps. For the first step, we have two options. The first is to prove a local result, where one only considers the dynamics in a small neighbourhood of a weakly (un)stable

*Institut für Mathematik, RWTH Aachen, Germany. The work of the first author was supported by DFG "Forschungsstipendium" grant BL 535/5-1. He would like to thank the IMA for support.

${ }^{\dagger}$ MRC, University of Warwick, UK. The work of the second author was supported by the Fonds National Suisse 
equilibrium. Here one can allow for blow ups of solutions far away from the equilibrium, but one then only gets estimates of the type "The rest term is small with high probability". If one takes into account stabilising properties of the nonlinearity, one can control all trajectories to get bounds on arbitrary $p$-th moments of the error term.

In the second step, one combines that approximation result with ergodic properties of the aproximating system to show that the long-time dynamics of the two systems are also close to each other. This is obviously only possible for nonlinear stable equations.

On finite domains a main assumption is the existence of a well-defined spectral gap separating the dominant modes that are subject to a weak instability or stability from the stable modes. In the deterministic setting, it is now well-known (e.g. [2]) how to derive invariant manifolds characterising the dominant flow of the equation. For the stochastic equation this is not clear. Recently the concept of random invariant manifolds for SPDE was developed (see [21] or [35]), but we are far from a complete understanding.

In this paper we take a different approach describing the flow around a fixed deterministic vector space spanned by the dominant eigenfunctions (or modes) of the linearisation. We neglect all the trajectories far away from that space, as they only have very small probability. This concept will be called approximate centre manifold.

This is in sharp contrast to the case of unbounded domains, where a whole band of modes gets unstable, and no spectral gap exists. For deterministic equations there exists a theory that replaces centre manifold theory (see [39] and the references therein). A similar situation occurs already if the size of the domain is of order $\varepsilon^{-1}$ for a noise and a linear instability of size $\varepsilon^{2}$ (see for example [33] for the unperturbed equation). For the stochastic equation this situation is currently under investigation in [9].

The paper is organised as follows. First, we state the general problem and make the formal multi-scale analysis in section 2 . In section 2.1, we then summarise all technical assumptions required for the proofs, and we give some examples of such equations in section 2.2. The main results for the approximation will be stated in section 3. In Section 4 we give the applications to approximate centre manifolds and random fixed points, while section 5 discusses approximation of invariant measures. The final section 6 gives a brief survey what results one expects for more general classes of nonlinearities, and we give a detailed discussion of the special case of quadratic nonlinearities.

2. General setting. We consider parabolic nonlinear SPDEs with additive forcing near a stability change, where the noise is of the order of the distance from the change of stability.

The general prototype is an equation of the type

$$
\partial_{t} u=L u+\varepsilon^{2} A u+\mathcal{F}(u)+\varepsilon^{2} \xi,
$$


where $L$ is a dissipative operator with finite dimensional kernel, $\varepsilon^{2} A u$ is a small (linear) deterministic perturbation, $\mathcal{F}$ is a nonlinearity, and $\xi=$ $\xi(t, x)$ is a Gaussian noise which will be taken to be white in time and can be either white or coloured in space. In other words, $\xi(t)=\partial_{t} Q W(t)$, where $W=\{W(t)\}_{t \geq 0}$ is a standard cylindrical Wiener process and $Q Q^{*}$ is the covariance operator (in space) of the noise. We will work with solutions in some Hilbert space $\mathcal{H}$.

One standard example of such an equation is given by the SwiftHohenberg equation

$$
\partial_{t} u=-(1+\Delta)^{2} u+\varepsilon^{2} u-u^{3}+\varepsilon^{2} \xi,
$$

with periodic boundary conditions on the domain $[0,2 \pi l]^{d}$ for dimension $d \in \mathbb{N}$ and integer length $l>0$. After slight modification we can also treat non-integer length $l>0$. For more examples see section 2.2.

Before we proceed to give detailed assumptions, we present a short formal derivation via multi-scale analysis of the amplitude equation, which was already discussed in [7]. Assume $\mathcal{F}$ to be cubic (i.e. trilinear) and denote the kernel of $L$ by $\mathcal{N}$. Denote the orthogonal projection onto $\mathcal{N}$ by $P_{c}$ and define $P_{s}=I-P_{c}$. Then we make the following ansatz:

$$
u(t)=\varepsilon a\left(\varepsilon^{2} t\right)+\varepsilon^{2} b\left(\varepsilon^{2} t\right)+\varepsilon^{2} \psi(t)+\mathcal{O}\left(\varepsilon^{3}\right),
$$

with $a, b, c \in \mathcal{N}$ and $\phi, \psi \in \mathcal{S}=P_{s} \mathcal{H}$. This ansatz is motivated by the fact that, due to the linear damping of order one in $\mathcal{S}$, the modes in $\mathcal{S}$ are expected to evolve on time scales of order one, whereas the modes in $\mathcal{N}$ are expected to evolve on much slower time scales of order $\varepsilon^{-2}$.

Plugging the ansatz (2.3) back into (2.1), setting $T=\varepsilon^{2} t$, and expanding in orders of $\varepsilon$, we obtain first, by collecting all terms of order $\varepsilon^{3}$ in $\mathcal{N}$

$$
\partial_{T} a(T)=A_{c} a(T)+\mathcal{F}_{c}(a(T))+\partial_{T} \beta(T) .
$$

Here, $\beta(T)=\varepsilon P_{c} Q W\left(\varepsilon^{-2} T\right)$ is a Wiener process in $\mathcal{N}$ with distribution independent of $\varepsilon$, and we write $A_{c}=P_{c} A$ and $\mathcal{F}_{c}=P_{c} \mathcal{F}$ for short. This approximation is called amplitude equation, and it is well-known for many examples in the physics and applied mathematics literature (e.g. [15, (4.31),(5.11)]). Moreover, there are numerous variants of this method. See for example [24]. For a modulation equation of highly oscillatory solutions see for example [28], but there are many more examples. However, these are all non-rigorous approximations using formal multi-scale analysis.

Let us now turn to higher order corrections. Collecting terms of order $\varepsilon^{2}$ in $\mathcal{S}$ yields for $\psi$ the linear equation

$$
\partial_{t} \psi(t)=L_{s} \psi(t)+P_{s} \xi(t),
$$

where we defined $L_{s}=P_{s} L$. One can show furthermore that $b=0$ (see [7] for a detailed argument). 
2.1. Assumptions. Let us summarise all assumptions necessary for our results. We do not focus on the highest possible level of generality, but stick to some simpler cases which cover all our examples.

Assumption 1. Let $\mathcal{H}$ be a separable Hilbert space and $\Delta$ (subject to some boundary conditions) be a selfadjoint version of the Laplacian on $\mathcal{H}$. Suppose $L=P(-\Delta)$ for some function $P$ such that $L$ is non-positive. Furthermore let the kernel $\mathcal{N}=\operatorname{ker} L$ of $L$ be non-empty and suppose $P(k) \rightarrow-\infty$ as $k \rightarrow \infty$.

We denote by $P_{c}$ be the orthogonal projection onto $\mathcal{N}$, which is finitedimensional by assumption on $P$. Define $\operatorname{dim}(\mathcal{N})=n \in \mathbb{N}$ and $P_{s}=1-P_{c}$. We also denote the range of $P_{s}$ by $\mathcal{S}$, so that $\mathcal{H}=\mathcal{N} \oplus \mathcal{S}$.

It is clear that there exists $\omega>0$ such that

$$
\left\|e^{t L}\right\|_{\mathcal{L}(\mathcal{H}, \mathcal{H})} \leq 1, \quad\left\|e^{t L} P_{s}\right\|_{\mathcal{L}(\mathcal{H}, \mathcal{H})} \leq e^{-t \omega} \quad \text { for any } t \geq 0
$$

We define the fractional space $\mathcal{H}^{\alpha}$ for $\alpha \geq 0$ as usual by $\mathcal{H}^{\alpha}=D((1-$ $L)^{\alpha}$ ) with norm $\|u\|_{\alpha}=\left\|(1-L)^{\alpha} u\right\|$. The space $\mathcal{H}^{-\alpha}=\left(\mathcal{H}^{\alpha}\right)^{\prime}$ is the dual of $\mathcal{H}^{\alpha}$ with respect to the pairing $\langle\cdot, \cdot\rangle$ (see for example [30] or [36]). It is wellknown that $e^{t L}$ extends to an analytic semigroup on all $\mathcal{H}^{\alpha}, \alpha \in \mathbb{R}$. Note furthermore that obviously $\mathcal{N} \subset \mathcal{H}^{\alpha}$ for any $\alpha \geq 0$, since $(1-L)^{\alpha} \mathcal{N}=\mathcal{N}$.

It is also a well-known fact that for $\alpha \in[0,1)$ there is a constant $M_{\alpha} \geq 1$ such that

$$
\left\|e^{t L}\right\|_{\mathcal{L}\left(\mathcal{H}^{-\alpha}, \mathcal{H}\right)} \leq M_{\alpha}\left(1+t^{-\alpha}\right)
$$

and for some $0<\tilde{\omega}<\omega$

$$
\left\|P_{s} e^{t L}\right\|_{\mathcal{L}\left(\mathcal{H}^{-\alpha}, \mathcal{H}\right)} \leq M_{\alpha}\left(1+t^{-\alpha}\right) e^{-t \tilde{\omega}} \quad \text { for all } \quad t>0
$$

Assumption 2. The function $\mathcal{F}$ is locally Lipschitz from $\mathcal{H}$ to $\mathcal{H}^{-\alpha}$ for some $\alpha \in[0,1)$. Assume we can split $\mathcal{F}(x)=f(x)+g(x)$, where $f: \mathcal{H} \times \mathcal{H} \times \mathcal{H} \rightarrow \mathcal{H}^{-\alpha}$ is continuous, trilinear, and symmetric. The function $g$ is higher order, which means $\|g(x)\|_{-\alpha} \leq C\|x\|^{4}$ provided $\|x\| \leq 1$.

Furthermore assume that $P_{c} f$ is stable on $\mathcal{N}$, which means $\mid P_{c} f(\varphi+$ $a) \cdot a \mid \leq C_{\delta}\|\varphi\|^{4}-\delta\|a\|^{4}$ for some small $\delta>0$ and all $a, \varphi \in \mathcal{N}$, where we use the canonical scalar product in $\mathcal{N} \approx \mathbb{R}^{n}$.

Finally, we assume $A: \mathcal{H} \rightarrow \mathcal{H}^{-\alpha}$ is a bounded linear operator.

We will use the shorthand notations $f(u)=f(u, u, u)$ and $\mathcal{F}_{c}=P_{c} \mathcal{F}$, Moreover $A_{c}=P_{c} A$ and $f_{c}=P_{c} f$.

In the following we make a somewhat stronger assumption ensuring global nonlinear stability of our SPDE (2.1). For simplicity, we restrict ourselves to cubic nonlinearities. This assumption is responsible for the global existence of solutions (cf. Proposition 2.1) and for uniform (in $t$ ) bounds on $\mathbb{E}\|u(t)\|^{p}$ for solutions of (2.1) (cf. Theorem 3.2). Note that these bounds are independent of the initial condition. 
Assumption 3. Let Assumption 2 be true and assume that the linear operator $A$ belongs to $\mathcal{L}\left(\mathcal{H}^{1}, \mathcal{H}\right)$. Moreover, there exists a constant $C_{A}>0$ such that

$$
\langle A v, v\rangle \leq C_{A}\left(\|v\|^{2}+\langle-L v, v\rangle\right) \quad \text { for all } \quad v \in \mathcal{H}^{1} .
$$

We also assume that $\mathcal{F}$ is trilinear, $\mathcal{F}:\left(\mathcal{H}^{1}\right)^{3} \rightarrow \mathcal{H}$ is continuous and that

$$
\left\langle\mathcal{F}_{c}\left(v_{c}, v_{c}, w_{c}\right), w_{c}\right\rangle<0
$$

for all $v_{c}, w_{c} \in \mathcal{N} \backslash\{0\}$. We finally assume that there exist constants $K$ and $\gamma_{L} \in[0,1)$ such that, for some $\delta>0$,

$$
\langle\mathcal{F}(v+\phi), v\rangle \leq K\|\phi\|^{4}-\delta\|v\|^{4}-\gamma_{L}\langle L v, v\rangle,
$$

for any $\phi, v \in \mathcal{H}^{1}$.

Concerning the stochastic perturbation we will always assume that the following is true. (For a detailed discussion of $Q$-Wiener processes and stochastic convolutions see [18].)

Assumption 4. The noise process is formally given by $\xi=Q \partial_{t} W$, where $W$ is a standard cylindrical Wiener process in $\mathcal{H}$ with the identity as a covariance operator and $Q \in \mathcal{L}(\mathcal{H}, \mathcal{H})$ is symmetric. Furthermore, there exists a constant $\tilde{\alpha}<\frac{1}{2}$ such that

$$
\left\|(1-L)^{-\tilde{\alpha}} Q\right\|_{\mathrm{HS}(\mathcal{H})}<\infty,
$$

where $\|\cdot\|_{\mathrm{HS}(\mathcal{H})}$ denotes the Hilbert-Schmidt norm of an operator from $\mathcal{H}$ to $\mathcal{H}$.

REMARK 2.1. Straightforward computations, combined with the properties of analytic semigroups allow to check that Assumption 4 implies the following (see [18, Section 5.4] for the first assertion):

- The stochastic convolution $W_{L}(t)=\int_{0}^{t} e^{L(t-s)} Q d W(s)$ is an $\mathcal{H}$ valued process with Hölder continuous sample paths.

- There exist positive constants $C$ and $\gamma$ such that

$$
\left\|P_{s} e^{L t} Q\right\|_{\mathrm{HS}} \leq C\left(1+t^{-\gamma}\right) e^{-\omega t},
$$

holds for every $t>0$.

REMARK 2.2. Note that we do not assume that $Q$ and $L$ commute. Hence, it is in general not true that $Q$ and $P_{c}$ commute. Therefore, the noise processes $P_{c} Q W$ and $P_{s} Q W$ will not necessarily be independent, which implies that the amplitude equation (2.4) and equation (2.5) for the second order correction are coupled through the noise.

It is straightforward to verify that

$$
P_{s}\left[W_{L}(t)\right]=\int_{0}^{t} e^{(t-\tau) L} d P_{s} Q W(\tau) \quad \text { and } \quad P_{c}\left[W_{L}(t)\right]=P_{c} Q W(t) .
$$


Therefore, the stochastic convolution is a Wiener process on $\mathcal{N}$ and it is a stable Ornstein-Uhlenbeck process on $\mathcal{S}$. This means that the noise acts in two completely different ways on $P_{c} u$ and $P_{s} u$ for some mild solution $u$.

To give a meaning to (2.1) we will always consider mild solutions, which are given by the following proposition.

Proposition 2.1. Under Assumption 1, 2, and 4, for any (stochastic) initial condition $u_{0} \in \mathcal{H}$ equation (2.1) has a unique local mild solution $u$. This means we have a stopping time $t^{*}>0$ and a stochastic process $u$ such that $u:\left[0, t^{*}\right] \rightarrow \mathcal{H}$ is a solution of

$$
u(t)=e^{t L} u_{0}+\int_{0}^{t} e^{(t-\tau) L}\left[\varepsilon^{2} A u+\mathcal{F}(u)\right](\tau) d \tau+\varepsilon^{2} W_{L}(t)
$$

for $t \leq t^{*}$.

Suppose additionally that Assumption 3 is true, then the solutions are global, which means $t^{*}=\infty$.

For the proof note that the existence and uniqueness of local solutions is standard since we consider locally Lipschitz-continuous nonlinearities. See for example [18, Section 7] we can also apply the deterministic approach of $\left[25\right.$, Thm. 3.3.3] path-wise. For $L^{p}$-theory with application to NavierStokes eq. see for example $[10,11]$.

The global existence follows from standard a-priori estimates for $v=$ $u-W_{L}$, as $v$ is a weak solution of the following PDE with random coefficients:

$$
\partial_{t} v=L v+\varepsilon^{2} A\left(v+W_{L}\right)+\mathcal{F}\left(v+W_{L}\right) .
$$

The formal idea is to take the scalar product with $v$, in order to derive estimates for $\|v\|^{2}$ and hence $\|u\|^{2}$.

2.2. Examples of equations. In the literature there are many examples of equations of the type given by Assumptions 1, 2 or 3, and 4. For instance, the well-known Ginzburg-Landau equation (see [20] for a standard proof of existence)

$$
\partial_{t} u=\Delta u+\nu u-u^{3}+\sigma \xi
$$

and the Swift-Hohenberg equation

$$
\partial_{t} u=-(\Delta+1)^{2} u+\nu u-u^{3}+\sigma \xi,
$$

which was first used as a toy model for the convective instability in a Rayleigh-Bénard problem (see [26] or [15]), fall into the scope of our work when the parameters $\nu$ and $\sigma$ are small and of comparable order of magnitude. Both equations are considered on bounded domains with suitable boundary conditions (e.g. periodic, Dirichlet, Neumann, etc.).

Other equations could involve nonlinearities of the type $\partial_{x}^{2}\left(u^{3}\right)$ or $\partial_{x}\left(\left(\partial_{x} u\right)^{3}\right)$. The first nonlinearity is considered with the Sobolev space 
$\mathcal{H}=H^{-1}$, while the second one has to be considered in $L^{2}$, provided we have the following Poincare type inequality $\|u\| \leq C\left\|\partial_{x} u\right\|$ for $u \in D(L)$.

Another example arising in the theory of surface growth is

$$
\partial_{t} u=-\Delta^{2} u-\mu \Delta u+\nabla\left(|\nabla u|^{2} \nabla u\right)+\sigma \xi,
$$

subject to periodic boundary conditions and moving frame $\int_{G} u d x=0$, which will ensure a Poincare type inequality. The deterministic equation was rigorously treated in [27]. Here we can consider $\mu=\mu_{0}+\varepsilon^{2}$ and $\sigma=\mathcal{O}\left(\varepsilon^{2}\right)$, where $\mu_{0}$ is such that $L=-\Delta^{2} u-\mu_{0} \Delta u$ fulfils Assumption 1 .

3. Amplitude equations, main results. We review the two main approaches to verify the approximation via amplitude equations. One relies on a purely local picture and uses Assumption 2, while the other takes into account the global nonlinear stability of the equation given by Assumption 3.

3.1. Attractivity. The attractivity justifies the ansatz for the formal computation. It shows that after a comparably short time the solution is of the form of the ansatz (2.3).

Theorem 3.1 (Attractivity-local). Under Assumptions 1, 2, and 4 there are constants $c_{i}>0$ and a time $t_{\varepsilon}=\mathcal{O}\left(\ln \left(\varepsilon^{-1}\right)\right)$ such that for all mild solutions $u$ of (2.14) we can write $u\left(t_{\varepsilon}\right)=\varepsilon a_{\varepsilon}+\varepsilon^{2} R_{\varepsilon}$ with $a_{\varepsilon} \in \mathcal{N}$ and $R_{\varepsilon} \in \mathcal{S}$, where

$$
\mathbb{P}\left(\left\|a_{\varepsilon}\right\| \leq \delta,\left\|R_{\varepsilon}\right\| \leq \varepsilon^{-\kappa}\right) \geq \mathbb{P}\left(\left\|u_{0}\right\| \leq c_{3} \delta \varepsilon\right)-c_{1} e^{-c_{2} \varepsilon^{-2 \kappa}},
$$

for all $\delta>0$ and $\varepsilon \in(0,1)$.

The proof of this result is a straightforward modification of Theorem 3.3 of [4]. It relies on the fact that small solutions of order $\mathcal{O}(\varepsilon)$ are on small time-scales given by the linearised picture, which is dominated by the semigroup estimates (2.7) and (2.8).

TheOREM 3.2 (Attractivity-global). Let Assumptions 1, 2, and 4 be satisfied. Then for all $T_{0}>0$ and $p \geq 1$ there are constants $c_{p}>0$ such that

$$
\mathbb{E}\left\|u\left(T_{0} \cdot \varepsilon^{-2}\right)\right\|^{p} \leq c_{p} \varepsilon^{p}
$$

for all $\mathcal{H}$-valued mild solutions $u$ of equation 2.1 independent of the initial condition.

Furthermore, there is a time $t_{\varepsilon}=\mathcal{O}\left(\ln \left(\varepsilon^{-1}\right)\right)$ such that given a family of positive constants $\left\{\delta_{p}\right\}_{p \geq 1}$ there are positive constants $\left\{C_{p}\right\}_{p \geq 1}$, such that for all $\mathcal{H}$-valued mild solutions $u$ of equation (2.1) with $\mathbb{E}\|u(0)\|^{p} \leq$ $\delta_{p} \varepsilon^{p}$ we have

$$
\mathbb{E}\|u(t)\|^{p} \leq C_{p} \varepsilon^{p} \quad \text { and } \quad \mathbb{E}\left\|P_{s} u\left(t+t_{\varepsilon}\right)\right\|^{p} \leq C_{p} \varepsilon^{2 p}
$$

for all $t \geq 0$ and $\varepsilon \in(0,1)$. 
The proof is given by a-priori estimates. This was not directly proved in [7], but under our somewhat stronger assumptions this is similar to Lemma 4.3 of [7]. It relies on a-priori estimates for $v_{\delta}=u-W_{L-\delta}$ with $\delta=\mathcal{O}\left(\varepsilon^{2}\right)$, which fulfils a random PDE similar to (2.15). We omit the proof, as it is technical but straightforward.

3.2. Approximation. For a solution $a$ of (2.4) and $\psi$ of (2.5) we define the approximations $\varepsilon w_{k}$ of order $k$ by

$$
\varepsilon w_{1}(t):=\varepsilon a\left(\varepsilon^{2} t\right) \text { and } \quad \varepsilon w_{2}(t):=\varepsilon a\left(\varepsilon^{2} t\right)+\varepsilon^{2} \psi(t) .
$$

The residual of $\varepsilon w$ is given by

$$
\begin{aligned}
\operatorname{Res}(\varepsilon w(t))= & -\varepsilon w(t)+e^{t L} \varepsilon w(0)+\varepsilon^{2} W_{L}(t) \\
& +\int_{0}^{t} e^{(t-\tau) L}\left[\varepsilon^{3} A w+\mathcal{F}(\varepsilon w)\right](\tau) d \tau .
\end{aligned}
$$

In order to show that $\varepsilon w$ is a good approximation of a solution $u$ of (2.14), the main step is to control the residual. The main idea is to obtain bounds on $P_{c} \operatorname{Res}(\varepsilon w)$ via the amplitude equation and to bound $P_{s} \operatorname{Res}(\varepsilon w)$ by using the stability of the equation which is ensured by our spectral gap (cf. (2.6) or (2.7)). These estimates require good a-priori bounds on the approximation $\varepsilon w_{k}$, but do not require any a-priori knowledge on the solution $u$ of the original equation.

Bounds on the residual easily imply approximation results, as we can establish bounds on the difference of $\varepsilon w_{k}$ and $u$ using (3.4) and (2.14).

Theorem 3.3 (Approximation-local). Suppose Assumptions 1, 2, and 4 are true. Fix the time $T_{0}>0$ and some small $\kappa \in(0,1)$. Then there are constants $C_{\text {att }}>0$ and $c_{i}>0$ such that for $\varepsilon \in(0,1)$ we obtain for all solutions $u$ of (2.14) and all solutions a of (2.4) (with $f_{c}$ instead of $\mathcal{F}_{c}$ )

$$
\begin{aligned}
& \mathbb{P}\left(\sup _{t \in\left[0, T_{0} \varepsilon^{-2}\right]}\left\|u(t)-\varepsilon w_{1}(t)\right\|_{X} \leq C_{\text {att }} \varepsilon^{2-\kappa}\right) \\
& \geq 1-\mathbb{P}\left(\left\|u_{0}-\varepsilon a(0)\right\|_{X} \geq c_{1} \varepsilon^{2-\kappa}\right)-\mathbb{P}\left(\left\|u_{0}\right\|_{X} \geq c_{2} \varepsilon\right)-c_{3} e^{-c_{4} \ln \left(\varepsilon^{-1}\right)^{2}} .
\end{aligned}
$$

The proof of this result is a straightforward modification of Theorems 4.1 and 4.3 of [4]. We use ideas of [9] to allow for weaker bounds on $\sup _{T \in\left[0, T_{0}\right]}|a(T)|$ by $c^{*} \ln \left(\varepsilon^{-1}\right)$, which were not present in [4] or [5]. There the probability was bounded by terms of order $o(1)$ in $\varepsilon$ without any further information on the smallness. Nevertheless, we can easily improve these proofs.

In that situation, we can use the following large deviation bound

$$
\mathbb{P}\left(\sup _{T \in\left[0, T_{0}\right]}|a(T)| \geq C \ln \left(\varepsilon^{-1}\right)\right) \leq C e^{-c \ln \left(\varepsilon^{-1}\right)^{2}},
$$


which is not exponentially small, but smaller than any power of $\varepsilon$. This relies on the fact that the amplitude equation is nonlinear stable, which follows from Assumption 3. This stability allows to carry over large deviation results for the Brownian motion $\beta$ in $\mathcal{N} \approx \mathbb{R}^{n}$ to results for $a$.

Under the stronger Assumption 3 we can prove a much better result.

Theorem 3.4 (Approximation-global). Let Assumptions 1, 3, and 4 hold and let $u$ be the mild solution of (2.1) with (random) initial value $u_{0}$ satisfying (3.3).

Then for all $p>0,1 \gg \kappa>0$ and $T_{0}>0$ there is a constant $C_{\text {app }}$ explicitly depending on $p$ and $T_{0}$ such that the estimate

$$
\mathbb{E}\left(\sup _{t \in\left[0, T_{0} \varepsilon^{-2}\right]}\left\|u(t)-\varepsilon w_{2}(t)\right\|^{p}\right) \leq C_{\mathrm{app}} \varepsilon^{3 p-\kappa}
$$

holds for $\varepsilon \in(0,1)$.

The proof is Corollary 3.9 of [7].

4. Applications. We give results for approximate centre manifolds and the dynamics of random fixed points.

4.1. Approximate Centre Manifold. This section uses the approximation results of the previous section. We rely especially on Theorem 3.4 to extend the results, which were briefly sketched in [4] or [5], by using second order corrections introduced in [7]. That is why we restrict ourselves to nonlinear stable equations given by Assumption 3.

Our main result will show that the flow along $\mathcal{N}$ is well approximated by the solution $a$ of the amplitude equation on a slow time scale. The distance from $\mathcal{N}$ is given by a fast oscillation $\psi$, which is a stationary Ornstein-Uhlenbeck process. And everything is valid only up to errors of order $\mathcal{O}\left(\varepsilon^{3-\kappa}\right)$ and with high probability.

The flow given by (2.1) is approximated with high probability as sketched in Figure 1. There the typical behaviour of solutions is given.

TheOREm 4.1. Suppose Assumptions 1, 3, and 4 are true, then there is a logarithmic time $t_{\varepsilon}=\mathcal{O}\left(\ln \left(\varepsilon^{-1}\right)\right)$ such that the following is true. For an arbitrary mild solution $u(t)$ of (2.1) with (random) initial condition $u_{0}$, such that $\mathbb{E}\left\|u_{0}\right\|^{p} \leq \delta_{p} \varepsilon^{p}$ for some fixed family of constants $\left\{\delta_{p}\right\}_{p \geq 1}$, we denote by $a(t)$ the solution of (2.4) with $a\left(t_{\varepsilon}\right)=\varepsilon^{-1} P_{c} u\left(t_{\varepsilon}\right)$. Furthermore let $\psi^{*}(t)$ be the stationary Ornstein-Uhlenbeck process solving (2.5) given by (4.1).

Finally, fix the time $T_{0}>0$, some small $\kappa \in(0,1)$, and any $p>0$. Then there exists a constant $C>0$ such that

$$
\mathbb{E}\left(\sup _{t \in\left[t_{\varepsilon}, T_{0} \varepsilon^{-2}\right]}\left\|u(t)-\varepsilon a\left(t \varepsilon^{2}\right)-\varepsilon^{2} \psi^{*}(t)\right\|_{X}^{p}\right)^{1 / p} \leq C \varepsilon^{3-\kappa}
$$

holds for $\varepsilon \in(0,1)$. 


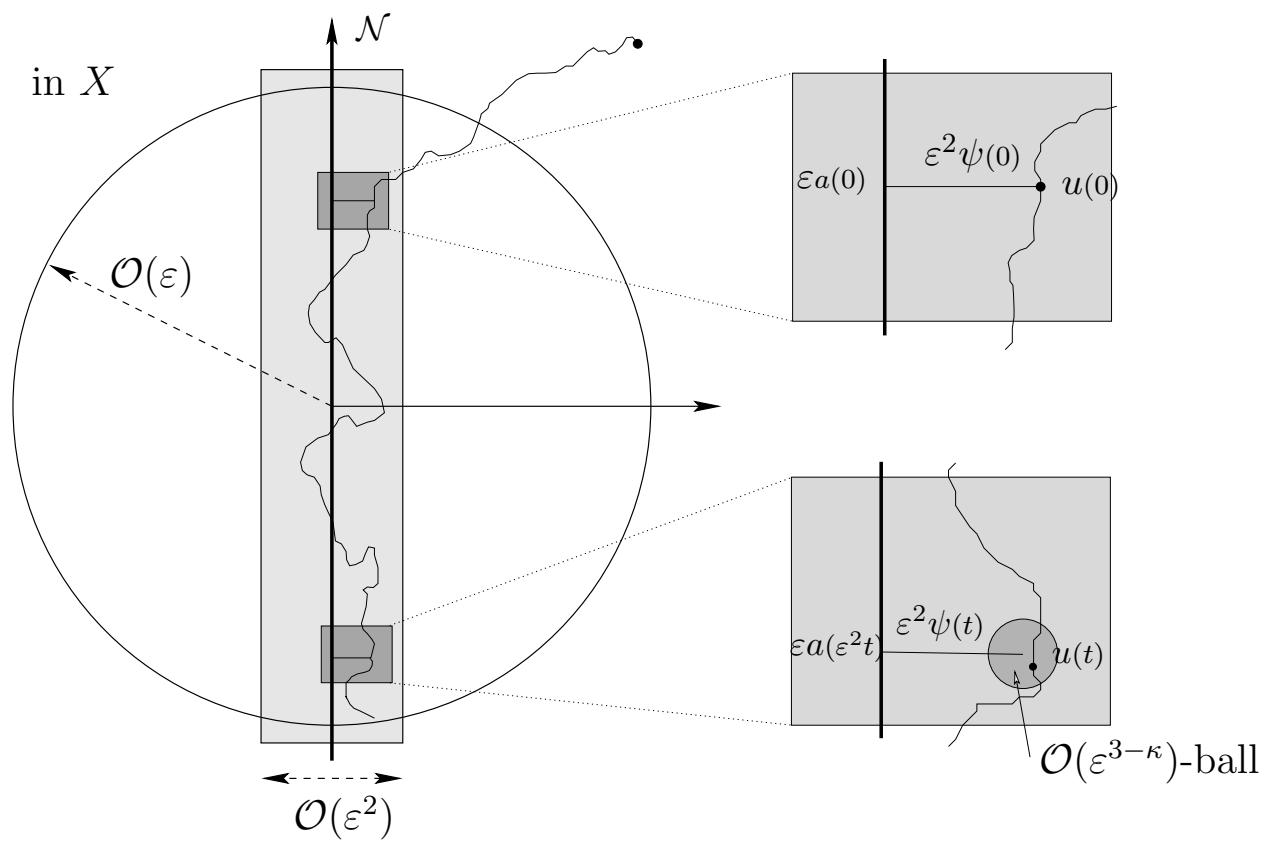

FIG. 1. Typical trajectory on the approximate centre manifold.

Proof. First we use global nonlinear attractivity in logarithmic time $t_{\varepsilon}^{(1)}$ for arbitrary initial conditions (cf. Theorem 3.2). Then we approximate with solution $\tilde{a}(t)$ of $(2.4)$ and $\tilde{\psi}(t)$ of $(2.5)$ for times $t \in\left[t_{\varepsilon}^{(1)}, T_{0} \varepsilon^{-2}\right]$. Define a version of the stationary Ornstein-Uhlenbeck process by

$$
\psi_{\star}(t)=\int_{-\infty}^{t} e^{-L(t-s)} d P_{s} Q \tilde{W}(s),
$$

where $\tilde{W}(s)=W(s)$ for $s>0$ and it is an independent Wiener process for $s<0$. For $\beta$ in the amplitude equation, we need only the rescaling $\beta(T)=\varepsilon P_{c} Q W\left(T \varepsilon^{-2}\right)$.

The difference between $\tilde{\psi}$ and $\psi^{*}$ is trivially small in any $p$-th moment, if we wait another sufficiently large logarithmic time $t_{\varepsilon}^{(2)}$. Define now $t_{\varepsilon}:=$ $t_{\varepsilon}^{(1)}+t_{\varepsilon}^{(2)}$.

The difference between $\tilde{a}(t)$ and $a(t)$ is small by the approximation result, because first $\left\|\tilde{a}\left(t_{\varepsilon}\right)-a\left(t_{\varepsilon}\right)\right\|=\mathcal{O}\left(\varepsilon^{3-\kappa}\right)$ by Theorem 3.4. Then, by the same theorem $\|\tilde{a}(t)-a(t)\| \leq\left\|\tilde{a}(t)-P_{c} u(t)\right\|+\left\|P_{c} u(t)-a(t)\right\|=$ $\mathcal{O}\left(\varepsilon^{3-\kappa}\right)$.

4.2. Dynamics of the random attractor. We can determine the dynamics of random fixed points by the approximation result over a very long time-scale with high probability. It suffices indeed to apply the results of the previous section by starting the equation in the random fixed point. 
Let us first fix some notation. If we consider a two sided Wiener process $W=\{W(t)\}_{t \in \mathbb{R}}$, then it is well known that solutions of $(2.14)$ define a random dynamical system (e.g. via transformation to (2.15)). Here $\varphi\left(t, u_{0}, W\right)$ is the solution $u(t)$ given initial condition $u_{0}$ and twosided noise path $W$. A random fixed point $a_{0}(W)$ is a random variable such that $\varphi\left(t, a_{0}(W), W\right)=a_{0}\left(\vartheta_{t} W\right)$, where $\vartheta_{t} W(s)=W(t+s)-W(t)$. For a detailed discussion of random dynamical systems see [1]. For the existence of random (set) attractors see for example [14, 40, 38].

Corollary 4.1. Under the assumptions of Theorem 4.1 let $a_{o}$ be a random fixed point of the random dynamical system generated by (2.14). Then

$$
\mathbb{E}\left(\sup _{t \in\left[t_{\varepsilon}, T_{0} \varepsilon^{-2}\right]}\left\|a_{o}\left(\vartheta_{t}\right)-\varepsilon a\left(t \varepsilon^{2}\right)-\varepsilon^{2} \psi^{*}(t)\right\|_{X}^{p}\right)^{1 / p} \leq C \varepsilon^{3-\kappa}
$$

where $a(0)=P_{c} a_{o}$, and $a$ is a solution of (2.4).

The proof is basically just a simpler case of Theorem 4.1. We start the system in the random fixed point $a_{0}$. In this case, we do not need time for attractivity, as due to the stationarity of $a_{0}$ and Theorem $3.2 u(0):=a_{0}$ already fulfils the assumptions of Theorem 3.4.

REMARK 4.1. We do not use uniformity in the initial condition. Hence, we can only prove results for random fixed points, and not for random set attractors but it would be an interesting result, whether we have

$$
\operatorname{dist}\left(P_{s} A\left(\vartheta_{t} \cdot\right), \psi^{*}(t)\right)=\mathcal{O}\left(\varepsilon^{3-\kappa}\right)
$$

on time intervals of order $\mathcal{O}\left(\varepsilon^{-2}\right)$ with high probability.

REMARK 4.2. The restriction to random fixed points still covers several cases. For example for dissipative nonlinearities $\varepsilon^{2} A+\mathcal{F}$ (e.g. $\left.-\varepsilon^{2} u-u^{3}\right)$ it is well known, that the random attractor is just a single random fixed point. For non-dissipative nonlinearities (e.g. $\left.\varepsilon^{2} u-u^{3}\right)$ it is in most cases completely open what the topology of the random attractor is. But, nevertheless, in many examples of non-trivial random attractors for SPDEs these attractors contain random fixed points.

If the attractor for the amplitude equation is a single stable fixed point $a^{*}$, which is exponentially attracting, then we can proof a much stronger result. We suppose the following.

Assumption 5. Suppose that the random dynamical system generated by the amplitude equation (2.4) has a unique random fixed point $a^{*}$ that is exponentially attracting in $p$-th mean. This means, for any $p>0$ there are constants $\delta>0$ and $M_{a}>0$ such that

$$
\mathbb{E}\left(\left\|a(T)-a^{*}\left(\vartheta_{T^{*}}\right)\right\|^{p}\right)^{1 / p} \leq M_{a} e^{-\delta T} \mathbb{E}\left(\left\|a(0)-a^{*}\right\|^{p}\right)^{1 / p}
$$

for any solution a of (2.4). 
For simplicity, we will rescale the equation to the slow time-scale $T=$ $\varepsilon^{2} t$. Consider the rescaling $v(T)=\varepsilon^{-1} u\left(T \varepsilon^{-2}\right)$, which is a solution of

$$
\partial_{T} v=\varepsilon^{-2} L v+A v+\mathcal{F}(v)+\partial_{T} Q \hat{W},
$$

where $\hat{W}$ is just a rescaling of $W$. Let $a$ be a solution of the amplitude equation with $\beta=P_{c} Q \hat{W}$ and let $\psi_{\varepsilon}$ be the rescaled Ornstein-Uhlenbeck process

$$
\psi_{\varepsilon}(t)=e^{\varepsilon^{-2} T L_{s}} \psi_{\varepsilon}(0)+\varepsilon^{-1} \int_{0}^{T} e^{-\varepsilon^{-2} L(T-s)} d P_{s} Q \hat{W}(s),
$$

Consider now the random dynamical system generated by the triple $\left(v, a, \varepsilon \psi_{\varepsilon}\right)$. It is obvious that random fixed points of this equation are just rescaled versions of random fixed points for the original system of equation. We can prove the following theorem.

TheORem 4.2. Suppose Assumptions 1, 3, 4, and 5 with random fixed point $a^{*}$ are true. Let $v^{*}$ be any fixed point of the rescaled equation (4.2) and denote by $\psi_{\varepsilon}^{*}$ the rescaled stationary $O U$-process. Then for any small $\kappa \in(0,1)$ and any $p>0$ there is a constant $C$ such that

$$
\mathbb{E}\left(\left\|v^{*}-a^{*}-\varepsilon \psi_{\varepsilon}^{*}\right\|_{X}^{p}\right)^{1 / p} \leq C \varepsilon^{2-\kappa} .
$$

Proof. For the proof consider first the projection to $\mathcal{N}$. Let $a$ be a solution of (2.4) with $\varepsilon^{-1} P_{c} v^{*}$. Using stationary

$$
\begin{aligned}
& \mathbb{E}\left(\left\|P_{c} v^{*}-a^{*}\right\|_{X}^{p}\right)^{1 / p} \\
& =\mathbb{E}\left(\left\|P_{c} v^{*}\left(\vartheta_{T} \cdot\right)-a^{*}\left(\vartheta_{T} \cdot\right)\right\|_{X}^{p}\right)^{1 / p} \\
& \leq \mathbb{E}\left(\left\|P_{c} v^{*}\left(\vartheta_{T} \cdot\right)-a(T)\right\|_{X}^{p}\right)^{1 / p}+\mathbb{E}\left(\left\|a(T)-a^{*}\left(\vartheta_{T} \cdot\right)\right\|_{X}^{p}\right)^{1 / p} \\
& \leq C \varepsilon^{2-\kappa}+M_{a} e^{-\delta T} \mathbb{E}\left(\left\|P_{c} v^{*}-a^{*}\right\|_{X}^{p}\right)^{1 / p}
\end{aligned}
$$

where we used the Approximation and Assumption 5. Given $p$ and $\kappa$, we can take $T$ sufficiently large such that $M_{a} e^{-\delta T}<\frac{1}{2}$. This yields the first part of the proof. The second part is completely analogous using the exponential stability of the Ornstein-Uhlenbeck process. $\mathrm{Q}$

REMARK 4.3. We can also establish an analog of the approximation result, which implies

$$
\mathbb{E} \sup _{t \in\left[0, T_{0}\right]}\left(\left\|v^{*}\left(\vartheta_{T} \cdot\right)-a^{*}\left(\vartheta_{T} \cdot\right)-\varepsilon \psi_{\varepsilon}^{*}\left(\vartheta_{T} \cdot\right)\right\|_{X}^{p}\right)^{1 / p} \leq C \varepsilon^{2-\kappa} .
$$

This is not an immediate consequence of a rescaled version of Theorem 3.4, we have to modify this slightly to allow for more general initial conditions for the amplitude equation. 
5. Approximation of the invariant measure. In this section, we review the results obtained in [7] on the invariant measure of (2.1). Recall that if $\Phi_{t}$ denotes the stochastic flow generated by the solutions to (2.1), then a measure $\mu_{\star}$ on $\mathcal{H}$ is called an invariant measure if

$$
\mu_{\star}(A)=\mathbb{E} \mu_{\star}\left(\Phi_{t}^{-1}(A)\right),
$$

for every $t>0$ and for every Borel set $A \subset \mathcal{H}$. For a general survey on invariant measures for SPDEs see [19].

The results of this section will rely on a very mild non-degeneracy condition for the stochastic forcing in (2.1) (cf. Assumption 4). This is the content of the next assumption. Note that this condition could be relaxed for some examples.

Assumption 6. The operator $P_{c} Q Q^{*} P_{c}$ is invertible as an operator from $\mathcal{N}$ to $\mathcal{N}$.

REMARK 5.1. This assumption ensures that the amplitude equation (2.4) has a unique invariant measure, and that this invariant measure has a $\mathcal{C}^{\infty}$ density with respect to the Lebesgue measure. Furthermore, we have exponential convergence of distributions of solutions to the invariant measure (see e.g. [32]).

There are many situations where this assumption also ensures the existence of a unique invariant measure for the original SPDE, see for example the recent works [29, 31, 22].

We will make use of two different norms to measure the distances between invariant measures. The first is the Wasserstein norm (also called Kantorovich distance), which is defined as the dual norm to the Lipschitz norm

$$
\|\phi\|_{\mathrm{L}}=\sup _{x, y \in \mathcal{H}}\left\{|\phi(x)|, \quad \frac{|\phi(x)-\phi(y)|}{\|x-y\|}\right\}
$$

i.e. one has

$$
\|\mu-\nu\|_{\mathrm{L}}=\sup _{\|\phi\|_{\mathrm{L}=\frac{1}{2}}}\left(\int \phi d \mu-\int \phi d \nu\right) .
$$

This will give us convergence for probabilities and, as we have uniform bounds on arbitrary moments (cf. Theorem 3.2), convergence of moments and other statistical quantities.

The second norm is the total variation norm $\|\cdot\|_{\mathrm{TV}}$, which is defined as the dual norm to the $L^{\infty}$-norm. Since $\|\phi\|_{\mathrm{L}} \geq\|\phi\|_{\infty}$, the total variation norm is stronger than the Wasserstein norm. Note that the Wasserstein norm depends strongly on the metric that equips the underlying space, whereas the total variation norm is independent of that metric. For example, the Wasserstein norm between two Dirac measures $\delta_{x}$ and $\delta_{y}$ is given by $\min \{1,\|x-y\|\}$, whereas $\left\|\delta_{x}-\delta_{y}\right\|_{\mathrm{TV}}$ is given by 1 if $x \neq y$ and 0 otherwise. (Actually, one can show that the total variation norm is equal to 
the supremum over all possible metrics of the corresponding Wasserstein norms. See [37] for a beautiful discussion of the relationship and properties between various metrics on probability measures.)

Before we state our results, we introduce one more notation. Similar to the proof of Theorem 4.2, we will rescale the solutions of (2.1) by $\varepsilon$ such that they are concentrated on a set of order 1 instead of a set of order $\varepsilon$. Furthermore, we will rescale the equation to the slow time-scale $T=t \varepsilon^{2}$.

We denote by $\mu_{\star}^{\varepsilon}$ the invariant measure of the rescaled version of (2.1). We furthermore denote by $\nu_{\star}^{\varepsilon}$ the invariant measure for the pair of processes $(a, \varepsilon \psi)$. Note that $\nu_{\star}^{\varepsilon}$ depends on $\varepsilon$ by the rescaling of $\psi$ and by the fact that equations (2.4) and (2.5) are coupled through the noise, but do not live on the same time scale. However, the marginal of $\nu_{\star}^{\varepsilon}$ on $\mathcal{N}$ is independent of $\varepsilon$ and its marginal on $\mathcal{S}$ depends on $\varepsilon$ only through the trivial scaling of $\varepsilon \psi$. We denote these two marginals by $\nu_{\star}^{c}$ and $\nu_{\star}^{s}$. With these notations, our result in the Wasserstein distance is the following:

Theorem 5.1. Let Assumptions 1, 3, 4, and 6 hold. Then, for every $\kappa>0$, one has

$$
\left\|\mu_{\star}^{\varepsilon}-\nu_{\star}^{c} \otimes \nu_{\star}^{s}\right\|_{\mathrm{L}}=\mathcal{O}\left(\varepsilon^{2-\kappa}\right) .
$$

REMARK 5.2. Actually, one also has $\left\|\mu_{\star}^{\varepsilon}-\nu_{\star}^{\varepsilon}\right\|_{\mathrm{L}}=\mathcal{O}\left(\varepsilon^{2-\kappa}\right)$, but the above formulation is more interesting, since $\nu_{\star}^{c}$ and $\nu_{\star}^{s}$ can be characterised explicitly, whereas $\nu_{\star}^{\varepsilon}$ can not, unless the covariance operator $Q$ is blockdiagonal with respect to the splitting $\mathcal{H}=\mathcal{N} \oplus \mathcal{S}$.

Idea of proof. Denote by $\mathcal{Q}_{t}$ the Markov transition semigroup (acting on measures) associated to the rescaled version of (2.1), and by $\mathcal{P}_{t}$ the transition semigroup associated to the evolution of $\left(a(t), \varepsilon \psi\left(\varepsilon^{-2} t\right)\right)$. Then, the main ingredient for the proof of Theorem 5.1 is that there exists a time $T$ such that, for every pair $(\mu, \nu)$ of probability measures with finite first moment, one has

$$
\left\|\mathcal{P}_{T} \mu-\mathcal{P}_{T} \nu\right\|_{\mathrm{L}} \leq \frac{1}{2}\|\mu-\nu\|_{\mathrm{L}}+\varepsilon^{2} \int_{X}\left(1+\left\|P_{s} x\right\|\right)(\mu+\nu)(d x) .
$$

In order to prove (5.5), one uses the strong contraction properties of the linear dynamic in $\mathcal{S}$ and that the strong mixing properties of the nondegenerate noise in $\mathcal{N}$.

Once (5.5) is established, the proof of Theorem 5.1 follows in a rather straightforward way. One first obtains from Theorem 3.4 that

$$
\begin{aligned}
\left\|\mu_{\star}^{\varepsilon}-\nu_{\star}^{\varepsilon}\right\|_{\mathrm{L}} & \leq\left\|\mathcal{Q}_{T} \mu_{\star}^{\varepsilon}-\mathcal{P}_{T} \mu_{\star}^{\varepsilon}\right\|_{\mathrm{L}}+\left\|\mathcal{P}_{T} \mu_{\star}^{\varepsilon}-\mathcal{P}_{T} \nu_{\star}^{\varepsilon}\right\|_{\mathrm{L}} \\
& \leq \mathcal{O}\left(\varepsilon^{2-\kappa}\right)+\frac{1}{2}\left\|\mu_{\star}^{\varepsilon}-\nu_{\star}^{\varepsilon}\right\|_{\mathrm{L}}+\mathcal{O}\left(\varepsilon^{2}\right),
\end{aligned}
$$

and therefore $\left\|\mu_{\star}^{\varepsilon}-\nu_{\star}^{\varepsilon}\right\|_{\mathrm{L}}=\mathcal{O}\left(\varepsilon^{2-\kappa}\right)$. The bound $\left\|\nu_{\star}^{\varepsilon}-\nu_{\star}^{c} \otimes \nu_{\star}^{s}\right\|_{\mathrm{L}}=\mathcal{O}\left(\varepsilon^{2-\kappa}\right)$ is then obtained by using the smoothness of the density of $\nu_{\star}^{c}$ with respect to 
the Lebesgue measure, combined with the separation of time scales between the dynamics on $\mathcal{N}$ and on $\mathcal{S}$.

The first result in the total variation norm only considers the marginals of the invariant measures on $\mathcal{N}$.

Theorem 5.2. Let Assumptions 1, 3, 4, and 6 hold. Then, for every $\kappa>0$, one has

$$
\left\|P_{c}^{*} \mu_{\star}^{\varepsilon}-\nu_{\star}^{c}\right\|_{\mathrm{TV}} \leq \mathcal{O}\left(\varepsilon^{\frac{3}{2}-\kappa}\right) .
$$

Idea of proof. We combine the smoothing properties of $P_{c} \mathcal{P}_{t} P_{c}$ with the result previously obtained in Theorem 5.1 to show that

$$
\left\|\mathcal{P}_{T} \mu_{\star}^{\varepsilon}-\mathcal{P}_{T} \nu_{\star}^{\varepsilon}\right\|_{\mathrm{L}} \leq \frac{C \varepsilon^{2-\kappa}}{\sqrt{T}} \quad \text { for all } \quad T \in(0,1] .
$$

Then, we use Girsanov's theorem to show that

$$
\left\|\mathcal{Q}_{T} \mu_{\star}^{\varepsilon}-\mathcal{P}_{T} \mu_{\star}^{\varepsilon}\right\|_{\mathrm{L}} \leq C \varepsilon \sqrt{T} \text { for all } T \in(0,1] .
$$

Combining both estimates and optimising for $T$ yields the result.

REMARK 5.3. The bound (5.6) is not always optimal. For example, when $L$ and $A$ are selfadjoint, $Q$ is the identity and $F$ is the gradient of a potential $V$, the rescaled invariant measure $\mu_{\varepsilon}^{\varepsilon}$ for (2.1) can formally be written as

$$
\mu_{\star}^{\varepsilon}(d u)=\exp \left(\frac{1}{2}\langle u, A u\rangle-V(u)\right) \mu_{0}^{\varepsilon}(d u),
$$

where $\mu_{0}^{\varepsilon}$ is the product of the Gaussian measure with covariance $\varepsilon^{2} L_{s}^{-1}$ on $\mathcal{S}$ and the Lebesgue measure on $\mathcal{N}$. This explicit expression allows one to show that the density of $P_{c}^{*} \mu_{\star}^{\varepsilon}$ has derivatives of all orders and that these derivatives are all of order 1 . This knowledge can be combined with Theorem 5.1 to show that in this case $\left\|P_{c}^{*} \mu_{\star}^{\varepsilon}-\nu_{\star}^{c}\right\|_{\mathrm{TV}}=\mathcal{O}\left(\varepsilon^{2-\kappa}\right)$. However, this argument fails completely if, for example, $P_{s} Q P_{s}=0$.

Our last result on the convergence of the invariant measures of the amplitude equation measures the distance between $\mu_{\star}^{\varepsilon}$ and $\nu_{\star}^{c} \otimes \nu_{\star}^{s}$ in the total variation norm. This however requires to impose a much stronger non-degeneracy assumption on the noise.

Assumption 7. Let $\alpha$ be as in Assumption 2. There exists a constant $\gamma_{0}>0$ such that, for all $\gamma \in\left[0, \gamma_{0}\right], \mathcal{F}:\left(\mathcal{H}^{\gamma}\right)^{3} \rightarrow \mathcal{H}^{\gamma-\alpha}$ and $A: \mathcal{H}^{\gamma} \rightarrow$ $\mathcal{H}^{\gamma-\alpha}$ are continuous. Furthermore, the operator $Q^{-1}$ is continuous from $\mathcal{H}^{\gamma_{0}-\alpha}$ to $\mathcal{H}$ and for some $\tilde{\alpha} \in\left[0, \frac{1}{2}\right)$ we have $\left\|(1-L)^{\gamma_{0}-\tilde{\alpha}} Q\right\|_{\operatorname{HS}(X)}<\infty$.

Theorem 5.3. Let Assumptions 1, 3, 4, and 7 hold. Then, for every $\kappa>0$, one has

$$
\left\|\mu_{\star}^{\varepsilon}-\nu_{\star}^{c} \otimes \nu_{\star}^{s}\right\|_{\mathrm{TV}}=\mathcal{O}\left(\varepsilon^{1-\kappa}\right) .
$$


Idea of proof. We denote by $\hat{\mathcal{P}}_{t}$ the transition probabilities associated to the linear system

$$
d u=\varepsilon^{-2} L u d t+Q d W(t)
$$

It is then possible to show as above by Girsanov theorem that

$$
\left\|\hat{\mathcal{P}}_{T} \mu_{\star}^{\varepsilon}-\mu_{\star}^{\varepsilon}\right\|_{\mathrm{TV}}=\left\|\hat{\mathcal{P}}_{T} \mu_{\star}^{\varepsilon}-\mathcal{Q}_{T} \mu_{\star}^{\varepsilon}\right\|_{\mathrm{TV}} \leq C \varepsilon^{-\kappa} \sqrt{T}+C \varepsilon
$$

Furthermore, the fast relaxation of the $\mathcal{S}$-component of the solutions to (5.11) toward its equilibrium measure, combined with the fact that the $\mathcal{N}$ marginals of $\mu_{\star}^{\varepsilon}$ and of $\nu_{\star}^{\varepsilon}$ are close by Theorem 5.2, allows to show that $\left\|\hat{\mathcal{P}}_{T} \mu_{\star}^{\varepsilon}-\nu_{\star}^{c} \otimes \nu_{\star}^{s}\right\|_{\mathrm{TV}} \leq C \varepsilon$, provided that $T \gg \varepsilon^{2}$. The result then follows by choosing $T$ of the order $\varepsilon^{2-\delta}$ for some small value of $\delta$.

6. What is so special about cubic nonlinearities? Cubic nonlinearities are not special, we can extend the method to a lot of different types of nonlinearities. Suppose we have a multi-linear nonlinearity, which is homogeneous of degree $n$. Then the noise strength in the SPDE (2.1) should be changed to $\varepsilon^{(n+1) /(n-1)}$ instead of $\varepsilon^{2}$. Now with the ansatz

$$
u(t)=\varepsilon^{2 /(n-1)} a\left(\varepsilon^{2} t\right)+\mathcal{O}\left(\varepsilon^{(n+1) /(n-1)}\right)
$$

and a similar formal calculation as in section 2, we derive the amplitude equation

$$
\partial_{T} a=P_{c} A a+P_{c} \mathcal{F}(a)+\partial_{T} \beta
$$

which now contains also a nonlinearity that is homogeneous of degree $n$.

We can verify this result rigorously. After minor changes the local theorems immediately carry over to these kinds of equations. For example for stable odd nonlinearities at least the order 1 approximation (local and global) is completely analogous.

The local approximation results also carry over to even nonlinearities, but one problem for global results is that we do not have nonlinear stability of the equations. In some cases, we can however get global results for even nonlinearities, if we already have good a-priori bounds for the solutions.

But the main problem with quadratic nonlinearities $B(u)=B(u, u)$ is that in many examples $P_{c} B(a) \equiv 0$ for $a \in \mathcal{N}$. In this case, the previously mentioned result will give us only the linearisation, meaning that we still look at solutions that are too small to capture the nonlinear features of the equation.

To illustrate this problem, we will briefly discuss Burgers equation, which is given by $\partial_{t} u=\partial_{x}^{2} u+\mu u-u \partial_{x} u+\sigma_{\varepsilon} \xi$.

For periodic boundary conditions and $\mu=\mathcal{O}\left(\varepsilon^{2}\right)$ we get $\mathcal{N}=\operatorname{span}\{1\}$ but now already $B(1)=0$. If we consider Dirichlet boundary conditions on $[0, \pi]$, for example, then the linear instability arises for $\mu=1+\mathcal{O}\left(\varepsilon^{2}\right)$. 
Furthermore, $\mathcal{N}=\operatorname{span}\{\sin \}$ and $B_{c}(\sin )=0$, where we used the shorthand notation $B_{c}=P_{c} B$ and $B_{s}=P_{s} B$.

There are numerous examples in the physics literature of equations with quadratic nonlinearities and the same property, as described above. One example is the growth of rough amorphous surfaces. See for example [6] and the references therein. Another example is the celebrated KuramotoSivashinsky equation, but the probably most important example is the Rayleigh-Bénard problem (see e.g. [23] or [15]) which is the paradigm of pattern formation in convection problems.

If we want to take into account nonlinear effects, we then have to look at the coupling of the slow dominant modes to the fast modes. This was done in [8] for the local result. Let us now briefly comment on these results.

Consider an equation of the type

$$
\partial_{t} u=L u+\varepsilon^{2} A u+B(u, u)+\varepsilon^{2} \xi
$$

with $B_{c}(a, a)=0$ for $a \in \mathcal{N}$, where $B$ is symmetric and bilinear. We make the ansatz

$$
u(t)=\varepsilon a\left(\varepsilon^{2} t\right)+\varepsilon^{2} \psi(t),
$$

with $a \in N(L)$ and $\psi \in P_{s} X$. This yields in lowest order in $\varepsilon$ the following system of formal approximations. First of order $\mathcal{O}\left(\varepsilon^{2}\right)$ on the fast timescale $t$ in $P_{s} X$.

$$
\partial_{t} \psi(t)=L_{s} \psi(t)+B_{s}\left(a\left(\varepsilon^{2} t\right), a\left(\varepsilon^{2} t\right)\right)+P_{s} \xi(t) .
$$

Secondly of order $\varepsilon^{3}$ in $N(L)$ on the slow time-scale $T=\varepsilon^{2} t$

$$
\partial_{T} a(T)=A_{c} a(T)+2 B_{c}\left(a(T), \psi\left(\varepsilon^{-2} T\right)\right)+P_{c} \hat{\xi}(T),
$$

where $\hat{\xi}(T)=\varepsilon^{-1} \xi\left(\varepsilon^{-2} T\right)$ is a rescaled version of the noise.

These equations are on one hand a dominating equation (6.2) on a slow time-scale coupled to an equation (6.1) on the fast time-scale. Equations with a similar structure are treated in [3] for stochastic ODEs, or in $[16,17]$ where tracers in a fast moving velocity field are considered. The aim is now to get an effective equation for the slow component completely independent of the fast modes.

First rescale (6.1) to the slow time-scale $T$ by $\psi(t)=\Phi\left(\varepsilon^{2} t\right)$. Hence,

$$
\varepsilon^{2} \partial_{T} \Phi(T)=L_{s} \Phi(T)+B_{s}(a(T), a(T))+\varepsilon P_{s} \hat{\xi}(T)
$$

As $L_{s}$ is invertible on $P_{s} X$, we get in lowest order of $\varepsilon$ that $\Phi(T)=$ $-L_{s}^{-1} B_{s}(a(T), a(T))$. This together with (6.2) establishes a single approximation equation.

$$
\partial_{T} a(T)=A_{c} a(T)-2 B_{c}\left(a(T), L_{s}^{-1} B_{s}(a(T), a(T))\right)+P_{c} \hat{\xi}(T)
$$


Surprisingly, this equation involves a cubic nonlinearity, although the nonlinearity in the original equation was quadratic.

The main results of [8] show that these formal calculations can be made rigorous in the sense of Theorems 3.1 and 3.3.

\section{REFERENCES}

[1] L. ARnold, Random dynamical systems, Springer Monographs in Mathematics. Springer, Berlin, 1998.

[2] B. Aulbach, Continuous and discrete dynamics near manifolds of equilibria, Lecture Notes in Math., 1058, Springer, Berlin, 1984.

[3] N. Berglund and B. Gentz, Geometric singular perturbation theory for stochastic differential equations, J. Differential Equations 191(1):1-54, (2003).

[4] D. Blömker, Amplitude equations for locally cubic non-autonomous nonlinearities, SIAM J. Appl. Dyn. Sys., 2(2):464-486, (2003).

[5] D. Blömker, S. Maier-PaApe, And G. Schneider, The stochastic Landau equation as an amplitude equation, Discrete and Continuous Dynamical Systems, Series B, 1(4):527-541, (2001).

[6] D. Blömker, C.GugG, M. Raible, Thin-film-growth models: roughness and correlation functions. European J. Appl. Math. 13(4):385-402, (2002).

[7] D. BlÖMker AND M. Hairer, Multiscale expansion of invariant measures for SPDEs, Submitted for publication, 2003.

[8] D. Blömker, Approximation of the stochastic Rayleigh-Bénard problem near the onset of instability and related problems, Submitted for publication, 2003.

[9] D. Blömker, M. Hairer, And G. Pavliotis, Stochastic amplitude equations in large domains, In Preparation, 2004.

[10] Z. Brzeźniak, S. Peszat, Space-time continuous solutions to SPDE's driven by a homogeneous Wiener process, Studia Math. 137(3):261-299, (1999)

[11] Z. Brzeźniak, S. Peszat, Strong local and global solutions for stochastic NavierStokes equations. Infinite dimensional stochastic analysis (Amsterdam, 1999), 85-98, Verh. Afd. Natuurkd. 1. Reeks. K. Ned. Akad. Wet., 52, R. Neth. Acad. Arts Sci., Amsterdam, (2000).

[12] P. Collet and J.-P. ECKMann, Instabilities and fronts in extended systems, Princeton Univ. Press, Princeton, NJ, 1990.

[13] P. Collet and J.-P. Eckmann, The time dependent amplitude equation for the Swift-Hohenberg problem, Comm. Math. Phys. 132(1):139-153, (1990).

[14] H. Crauel, A. Debussche, and F. Flandoli, Random attractors, J. Dynam. Differential Equations, 9(2):307-341, (1997).

[15] M. C. Cross And P. C. Hohenberg, Pattern formation outside of equilibrium, Rev. Mod. Phys. 65:851-1112, (1993).

[16] G. Pavliotis, A. Stuart. White noise limits for inertial particles in a random field. Preprint, (2003).

[17] G. Pavliotis and A. Stuart. Itô versus Stratonovich white noise limits. Preprint, (2003).

[18] G. Da Prato and J. Zabczyk, Stochastic Equations in Infinite Dimensions, Cambridge University Press, 1992.

[19] G. Da Prato And J. Zabczyk, Ergodicity for infinite-dimensional systems, London Mathematical Society Lecture Note Series, 229, Cambridge University Press, (1996).

[20] J. Duan, V.J. ERvin, On nonlinear amplitude evolution under stochastic forcing, Appl. Math. Comput. 109(1):59-65, (2000).

[21] J. Duan, K. Lu, and B. Schmalfuss, Invariant manifolds for stochastic partial differential equations, The Annals of Probability, to appear.

[22] W. E AND D. LiU, Gibbsian dynamics and invariant measures for stochastic dissi- 
pative PDEs, Journal of Statistical Physics, 108:4773-4785, (2002)

[23] A.V. Getuing Rayleigh-Bénard Convection - Structures and Dynamics. World Scientific Press, 1998.

[24] H. HAKEn, Synergetics. An introduction. Nonequilibrium phase transitions and self- organization in physics, chemistry, and biology, Springer Series in Synergetics, Vol. 1. Berlin etc.: Springer, 1983.

[25] D. Henry, Geometric theory of semilinear parabolic equations, Lecture Notes in Mathematics, 840. Berlin etc.: Springer, 1981.

[26] P.C. Hohenberg AND J.B. Swift, Effects of additive noise at the onset of Rayleigh-Bénard convection, Physical Review A, 46:4773-4785, (1992).

[27] B.B. King, O. Stein, And M. Winkler, A fourth-order parabolic equation modeling epitaxial thin film growth, J. Math. Anal. Appl. 286(2):459-490, (2003).

[28] R. Kuske, Multi-scale analysis of noise-sensitivity near a bifurcation, Proceedings of the IUTAM Symposium held in Monticello, IL, USA, 26-30 August 2002 (eds. N. Sri Namachchivaya and Y.K. Lin), Kluwer, 2002.

[29] S. B. Kuksin and A. Shirikyan, A Coupling Approach to Randomly Forced Nonlinear PDE's. I, Commun. Math. Phys., 221:351-366, (2001).

[30] A. LunARd, Analytic semigroups and optimal regularity in parabolic problems, Progress in Nonlinear Differential Equations and their Applications, 16. Birkhäuser Verlag, Basel, 1995.

[31] J. C. Mattingly, Exponential convergence for the stochastically forced NavierStokes equations and other partially dissipative dynamics, Commun. Math. Phys., 230:421-462, (2002).

[32] S. P. Meyn And R. L. Tweedie, Markov Chains and Stochastic Stability, Springer, New York, 1994.

[33] A. Mielke, G. Schneider, And A. Ziegra, Comparison of inertial manifolds and application to modulated systems, Math. Nachr., 214:53-69, (2000).

[34] A. Mielke And G. Schneider, Attractors for modulation equations on unbounded domains - existence and comparison, Nonlinearity, 8:743-768, (1995).

[35] S. Mohammed, T. Zhang, and H. Zhao, The Stable Manifold Theorem for Semilinear Stochastic Evolution Equations and Stochastic Partial Differential Equations. Part I: The Stochastic Semiflow, preprint, (2003).

[36] A. PAZY, Semigroups of Linear Operators and Application to Partial Differential Equations, Springer, 1983.

[37] S. Rachev, Probability metrics and the stability of stochastic models, John Wiley \& Sons Ltd., Chichester, 1991.

[38] M. Scheutzow, Comparison of various concepts of a random attractor: a case study, Arch. Math. (Basel),78(3):233-240, (2002).

[39] G. Schneider, Bifurcation theory for dissipative systems on unbounded cylindrical domains - an introduction to the mathematical theory of modulation equations, ZAMM (Z. Angew. Math. Mech.) 81(8):507-522, (2001).

[40] B. Schmalfuss, Measure attractors and random attractors for stochastic partial differential equations, Stochastic Anal. Appl., 17(6):1075-1101, (1999). 